\title{
Colegio y Universidad de La Plata: aportes de Joaquín V. González en el contexto de fundación de la universidad platense
}

\author{
Colegio Nacional and Universidad Nacional de La Plata: \\ Joaquin V. González's contributions in the establishment \\ of the university in La Plata
}

\author{
Juan Jowen Pratto
}

Orcid: https://orcid.org/0000-0002-3835-6518

Departamento de Educación Física, Universidad Nacional de La Plata, Río Grande, Tierra del Fuego, Argentina, jowenpratto@gmail.com

Received on 10/05/2020 - Approved on 20/05/2020

\section{Resumen}

El siguiente trabajo presenta algunos aportes del Dr. Joaquín V. González, Ministro de Justicia e Instrucción Pública (1904-1906), fundador de la Universidad Nacional de La Plata. En el recorrido se trabajará la historia del Colegio Nacional de La Plata y su pasaje a la Universidad; el contexto de la Educación Física nacional; mientras que también se hará mención especial a la idea y concepción de vida higiénica y ejercicios físicos entendida por el ministro riojano, tanto para el Colegio, como para la Universidad. Su nueva visión implicó grandes reformas tanto en la infraestructura como a nivel ideológico. Especial atención tendrá el nuevo concepto de Universidad abierta e Internado en el cual, las prácticas corporales y juegos al aire libre tendrán un papel fundamental.

Palabras clave: Colegio. Universidad. Internado. La Plata. Ejercicios Físicos. Educación Física.

\footnotetext{
Abstract

This paper addresses some of the contributions of Joaquin V. González, Minister of Justice and Public Instruction (1904-1906) and founder of the National University of La Plata. Starting with an overview of the history of the National School and its inclusion as part of the University, the paper explores the importance of Physical Education in a context of nationalization and how the Minister conceived and developed his idea of students leading a physical and
} 
hygienic life, both in the school and the university. Gonzalez's vision was innovative and reflected a new way of seeing physical education, which resulted in major changes. Since the National University had an ideological framework different from other universities, the paper will also focus on how it tried to distance itself from a conservative order to include students from different backgrounds through, among others, a boarding school, corporal practices and outdoor games.

Keywords: School. University. Boarding school. La Plata. Physical exercise. Physical education.

\section{Introducción}

Como ya se sostuvo en el resumen, el siguiente trabajo intenta rescatar la figura del Dr. Joaquín V. González quien, desde su cargo de Ministro de Justicia e Instrucción Pública de la Nación en 1905 bajo la presidencia de Manuel Quintana (1904-1906), funda la Universidad Nacional de La Plata, institución que tiene sus orígenes en una serie de institutos científicos y la decadente Universidad de La Plata. La casa de estudios platense fue creada bajo la órbita provincial en 1889 a raíz de un proyecto de ley provincial, encabezado por el legislador Rafael Hernández. El escrito también recorre la creación del Colegio Provincial fundado en 1885 y el pasaje del Colegio y la Universidad a la Nación, en 1887 y 1905 respectivamente. También se hará mención a la propuesta de reforma íntegra de la casa de estudios propuesta por González y la importancia que le dio a las prácticas corporales, juegos y deportes tanto para el Colegio como para la Universidad a través de la creación del campo de juegos atléticos, único en su tipo, en 1910.

\section{El ocaso de Buenos Aires y el amanecer de La Plata}

La federalización de la ciudad de Buenos Aires y la fundación de la ciudad de La Plata (nueva capital de la provincia de Buenos Aires) dieron por terminado un largo enfrentamiento entre facciones acerca de la organización política de la nación. Luego de la caída de Rosas, en 1852, se pretendió sancionar una Constitución para poder dar una organización política y comenzar a sentar las bases pero Buenos Aires, celosa de su autonomía y sus riquezas a través de la posesión del puerto y la aduana, siempre se negó. Ante las reiteradas diferencias, la provincia se constituyó en un estado separado de la Confederación Argentina, por lo que hasta finales de la década de 1850, se la conoce como el período de secesión. El estado autónomo sancionó su Constitución, negando así la que había sido firmada por las demás provincias cuando constituyeron la Confederación Argentina (gobernada desde 1854 por Justo José de Urquiza y desde 1860 por Santiago Derqui).

La relación del Estado de Buenos Aires y la Confederación fue siempre conflictiva y dentro de la provincia se conformaron facciones políticas que abogaban por la autonomía y otros que, al contrario, buscaban la unión 
nacional. Los conflictos se agudizaron por lo que llevaron a un enfrentamiento armado entre los generales Mitre (Buenos Aires) y Urquiza (Confederación) en la batalla de Cepeda en 1859. Finalizada la contienda armada, se firma un nuevo pacto de paz que vuelve a caer dos años después, en 1861. En la batalla de Pavón, Mitre se enfrenta al presidente Derqui (repudiado por Mitre por el fusilamiento del gobernador de San Juan), triunfando esta vez las fuerzas de Buenos Aires. Mitre toma el control de la Confederación y comienza un largo proceso de anexión de Buenos Aires a la Nación dando fin a la secesión. Hasta 1880, la provincia y la nación tuvieron varios enfrentamientos políticos y militares que recién se saldaron sobre el final de la presidencia de Avellaneda.

La década de 1880 es entendida por los historiadores como el punto de llegada del largo proceso de formación del Estado nacional el cual, según Hilda Sabato (2012), "logró subordinar a la última provincia con arrestos de autonomía, fortaleciendo así su organización y sus instituciones" mientras que la sanción de una ley que prohibió a las provincias mantener cuerpos militares, "fue un paso definitivo en la consolidación estatal, pues confirmó el monopolio de la fuerza por parte de la autoridad nacional. (p. 286).

Una vez federalizada la ciudad de Buenos Aires, (la última resistencia local que fue aniquilada por la gran figura política y militar que emergió en esta nueva década: Julio Argentino Roca), junto con su puerto y aduana, por supuesto, y el debido traspaso de las tierras y las instituciones que poseía al Estado Nacional, la provincia más poderosa política y económicamente perdía, entre otras cosas, su Universidad que había sido creada en 1821 bajo el gobierno del Martín Rodríguez. Por otro lado, al asumir el gobierno el Dr. Dardo Rocha (quien llegó a la gobernación gracias al apoyo de Roca) en 1881, se propuso la fundación de una nueva ciudad que albergaría las dependencias administrativas de la Provincia de Buenos Aires, así comenzó la carrera de la "Cuestión Capital". Rocha, secundado por Adolfo González Chaves, asume la gobernación el 1 de mayo de 1881 y rápidamente pone manos a la obra con tres temas prioritarios cuasi obsesivos: "dotar a Buenos Aires de una capital, devolverle a la provincia la grandeza que históricamente ha tenido y acceder a la presidencia de la nación." (Troisi, 2006: 69).

A poco más de un año y en tiempo récord, hechos los análisis pertinentes de suelos, accesibilidad, distancia de la ciudad de Buenos Aires, etc., de distintos lugares ofrecidos para albergar la capital (ciudades como Quilmes, Las Flores o Chivilcoy), y nuevas tierras para la construcción de la nueva ciudad, finalmente se eligió la zona de las lomas de Ensenada para la fundación de la ciudad de La Plata. El 19 de noviembre de 1882 se coloca la piedra fundacional de la ciudad higiénica por excelencia, cruce entre positivismo, ciencia, higienisimo, masonería y racionalidad (Vallejo, 2007). Por diferencias políticas que ya eran insalvables, el presidente Roca no asistió al acto de colocación de la piedra fundacional, excusándose de tener que realizar un viaje "impostergable" al interior del país. En su lugar envió al ministro de Relaciones Exteriores e interino del Interior, el Dr. Victorino de la Plaza ya que, por razones de salud, tampoco pudo asistir el vicepresidente de la nación, Francisco Madero. Si bien Rocha fue uno de los principales aliados de Roca, su afán modernizador, su carrera vertiginosa en la "cuestión Capital" para fundar La Plata, y su intención de utilizar la gobernación de Buenos Aires como 
un «trampolín» para llegar a la primera magistratura nacional, fueron los principales factores que marchitaron la relación con el presidente, quien en un principio, sino se convirtió en un enemigo, al menos se alejó bastante. Ya a fines de ese año, en 1882, Roca desconfiaba de su antiguo aliado, sosteniendo que en Rocha habitaba aún el espíritu del rebelde Tejedor. Incluso ve en Rocha "la principal amenaza a su poder; una vez en la presidencia, el acceso a los recursos nacionales y provinciales le otorgaría un poderío difícil de controlar que no le permitiría jamás a Roca el reingreso a la presidencia." (Troisi, 2006: 43). Ello llevó a Roca a comenzar a tejer las alianzas para la sucesión presidencial para cual fue elegido su concuñado, el Dr. Miguel Juárez Celman, quien llegará a la presidencia en 1886.

La ciudad de La Plata se concibió y diseñó en los papeles antes de poner el primer ladrillo. Su traza original, desarrollada por el Departamento de Ingenieros, con Pedro Benoit a la cabeza y con aportes del arquitecto Juan Burgos, terminan enviando el proyecto de plano el cual tiene la forma de un cuadrado perfecto, con una plaza principal en su centro geográfico, y dos diagonales que lo atraviesan de norte a sur y de este a oeste. El plano se basa en una concepción racionalista basado en preceptos de la higiene, la ciudad contó con un bosque propio, amplias avenidas, plazas y parques ubicados exactamente cada 6 cuadras en los cruces de avenidas, y el acceso al puerto natural de Ensenada. La ciudad contó con un eje cívico principal en los cuales se manifestó "la representación del poder" (Vallejo, 2007), desde la Avenida 1 hasta la calle 15 y limitado por las Avenidas 51 y 53, a través de la implantación de los edificios públicos como Comisaría de Policía (hoy Ministerio de Seguridad de la Provincia de Buenos Aires), Casa de Gobierno, Legislatura Provincial, Municipalidad y Templo. ${ }^{1}$ A ambos lados del eje principal se establecieron de modo equidistante de la plaza principal (hoy Plaza Moreno), el Palacio de Justicia (Avenida 13 entre calles 47 y 48), Consejo de Educación (hoy Dirección General de Escuelas de la Provincia de Buenos Aires, Avenida 13 entre calles 56 y 57). Mientras que a ambos lados de la Plaza San Martín (sobre la Avenida 7), se instalaron los Ministerios de Hacienda (Avenida 7 entre calles 45 y 46) y de Gobierno (hoy Ministerio de Infraestructura y Servicios Públicos, Avenida 7 entre calles 58 y 59).

\section{La situación escolar en La Plata}

En el presente apartado se hará un breve repaso de la situación escolar luego de fundada la ciudad, para luego desarrollar la creación del colegio secundario que, como veremos, primero se creó bajo la órbita provincial para luego pasar a la tutela de la Nación.

Con respecto a la educación primaria, y teniendo en cuenta que desde que se colocó la piedra fundacional de La Plata en noviembre de 1882 con lo cual se movilizaron grandes cantidades de obreros con sus familias para "levantar" la ciudad, el gobierno provincial se encargó rápidamente de la situación importando casillas de madera directamente desde los Estados Unidos para albergar a los pequeños estudiantes y los docentes. A medida que la construcción de los edificios públicos y la ciudad avanzaba, aquellas casillas 
de madera fueron cediendo antes los Palacios de la Educación, que ya tenían lugar en los planos de la ciudad en diversas manzanas y medias manzanas de la traza original. Los mismos fueron diseñados por el arquitecto Carlos Altgelt luego de su estadía en Alemania donde cursó sus estudios universitarios. Al volver al país, Altgelt se convirtió rápidamente en un referente de la arquitectura escolar, ocupando diferentes cargos en la Dirección General de Escuelas de la Provincia y en distintos Consejos Escolares. En el caso platense se le asignó el diseño y construcción del Palacio de la Educación (actual Dirección General de Cultura y Educación, sito en calle 13 entre 56 y 57 ) cuya piedra fundacional se constituyó en noviembre de 1883 contando con la presencia ni más ni menos que el propio gobernador Dardo Rocha, el Presidente del Consejo Nacional de Educación, Dr. Benjamín Zorrilla, el Director General de Escuelas y Presidente del Consejo General de Educación de la Provincia, Dr. Nicolás Achaval, el vicepresidente del Consejo General de Educación, Dr. Juan Manuel Ortiz de Rozas ${ }^{2}$, y el Dr. Eduardo Wilde, Ministro de Justicia, Culto e Instrucción Pública de la Nación entre otras destacadas personalidades. A su vez, los diferentes Templos de la educación se fueron construyendo gracias a las partidas presupuestadas asignadas por Rocha primero, continuado por Carlos D'amico (gobernador 1884-1887) y luego por Máximo Paz (1887-1890).

La situación de la educación secundaria en La Plata fue más compleja, ya que habiéndose entregado la ciudad de Buenos Aires a la Nación, la provincia perdió su Colegio Nacional y la Universidad. Para colmo de males, la Constitución Provincial vigente, de 1873, dispuso que la educación secundaria dependiera exclusivamente de la universidad, por lo que la educación secundaria se encontraba en una encrucijada, ya que no tenía lugar. El hecho se agravó en 1883 cuando el gobierno provincial sancionó una ley de residencia, en la cual obligaba a trasladarse a La Plata a todos los funcionarios públicos con sus familias: el gobernador y sus ministros; miembros de la Suprema Corte de Justicia, vocales de la cámara de apelaciones; jueces y todo el personal de la Administración del departamento judicial; el Director General de Escuelas y todos los consejeros de educación; todos aquellos empleados de las reparticiones públicas cuyas funciones se requiera que se den en la nueva Capital y, por último, los jubilados y pensionados de la Provincia.

El problema radicó en que aquellos funcionarios públicos debían enviar a sus hijos, en edad de cursar la educación secundaria, al Colegio Nacional de Buenos Aires u otros colegios particulares como los colegios de San José, el Literario, o el Británico (colegios que concentraban gran cantidad de estudiantes), de la ciudad de Buenos Aires. En este caso, los jóvenes debían instalarse en internados como pupilos o viajar desde La Plata a través del tren diario que partía desde la Estación Ferroviaria "19 de Noviembre", ubicada en la manzana comprendida por las calles $7,49,6$ y 50 frente a la Plaza San Martín (actualmente, en el ex edificio ferroviario, se encuentra el Pasaje Cultural Dardo Rocha).

Según Gustavo Vallejo (2007), Rocha y sus funcionarios rápidamente dieron batalla en dos frentes para poder contar con un colegio secundario: por un lado, gracias a un proyecto del diputado nacional por la Provincia de Buenos Aires, Manuel Lainez, se presentó un proyecto para la apertura de un Colegio Nacional similar al de la capital nacional; la ley de creación del Colegio de La 
Plata fue promulgada en julio de 1884. En el otro frente de batalla el mismo Rocha, secundado por su Ministro de Gobierno Carlos D'amico (quien sería gobernador en el período siguiente 1884-1887), realizaron un proyecto de educación de tipo práctica e industrial: el "Colegio Modelo de Educación Práctica". Para el desarrollo del mismo encargaron a Paul Groussac ${ }^{3}$ un estudio de establecimientos europeos similares. El proyecto es elevado a la legislatura provincial pero cae rápidamente al conocerse la ley de creación del Colegio Nacional de La Plata de 1884. La idea de crear un colegio de tipo "práctico" le costó a Rocha, ya que la misma no sería gratuita, sino costeada por sus propios alumnos; al conocerse en la cámara de diputados de la provincia que el Colegio Nacional para la ciudad ya había sido aprobado, "¿Para qué quería semejante colegio secundario la capital de la provincia si ya iba a tener uno de la misma índole creado por la Nación, con la enorme ventaja para este último de que él sería de enseñanza gratuita?" (Blanco, 1940/41: 118). De esta manera y con otras voces en contra, se desestima el proyecto confiando en la creación del Colegio Nacional.

La fundación de esta institución no sería fácil, ya que primero el Ministro de Instrucción Pública de la Nacion, Dr. Eduardo Wilde, solicitó una evaluación para implantar el colegio; la misma fue encomendada al mismísimo Paul Groussac (quien aceptó el encargo de Rocha pero que luego de pasado un tiempo nunca realizó el informe para la institución modelo de educación práctica), quien ocupaba el cargo de Inspector de Enseñanza Secundaria y Normal. Groussac viaja a La Plata, recorre las escuelas, se entrevista con el Consejo Escolar y luego se retira rápidamente a la capital. Su informe fue deliberada-mente negativo, fundamentando que no había alumnos para justificar la apertura de un colegio secundario, con ese dictamen, la Nación aplazó la fundación. El inspector se basó en los informes del Consejo y las estadísticas del año 1884 en la cual, realmente, había un número menor de niños que pudieran cursar sus estudios en el colegio secundario; pero ello se dio ya que todavía no había sido efectivizada la ley de residencia que impuso la legislatura el año anterior, que recién sería ejecutada realmente por el gobernador D'amico, cuando en 1884 se trasladan finalmente las autoridades provinciales a La Plata (menos de un mes antes de dar entregar la provincia a D'amico, Rocha se asienta en la casa de Gobierno en La Plata). La ley de creación del Colegio Nacional preveía esta situación, por ello mismo fue fundada; por otro lado, la creación se fundaba en que era la única de las 14 provincias que no contaba con Colegio Nacional y, por sobre todas las cosas, La Plata era la capital de la provincia más rica y poderosa

La realidad debemos leerla entre líneas ya que detrás de los números y las estadísticas siempre fue una cuestión macropolítica que, en todo caso, fue disfrazada con los números. Entre 1884 y 1885 ya estaba a pleno la carrera por la sucesión presidencial y, como comenté más arriba, Roca y Rocha ya eran enemigos insalvables. Quizás ingenuamente se lo ha tildado a Groussac y su informe como los culpables de la negativa a la fundación del Colegio Nacional pero, en realidad, deberíamos pensar que el Inspector actuó presionado por el Ministro Wilde, quien a su vez seguía las instrucciones del presidente Roca. Según Marcos Blanco, es Roca el principal artífice del desconocimiento de la ley de creación del Colegio, y ello solo puede ser entendido en línea de la política nacional. Por supuesto que el informe fue repudiado por el gobierno de 
la provincia, especialmente por el Director General de Escuelas Juan Manuel Ortiz de Rozas quien sostenía que:

Sobre un campo desierto nos pusimos a levantar apresuradamente casillas que se llenaron de niños antes de concluidas; hoy llegan a doce las escuelas y hay educandos para llenar veinte más [...] otro tanto va a suceder con el Colegio Nacional: instálese y se verá inmediatamente cien niños en sus aulas. (Ortiz de Rosas, 1884, en Vallejo, 2007: 111).

\section{Los Colegios Nacionales y la Educación Física}

Primero comentaré a grandes rasgos el origen de los Colegios para luego centrarme en la Educación Física. El sistema de Colegios Nacionales se implantó en 1863 bajo la presidencia de Bartolomé Mitre (1862-1868), el primer Colegio fundado fue el de Buenos Aires, luego de un encargar una comisión presidida por el francés Amadeo Jacques ${ }^{4}$ la cual tenía que elaborar una memoria para redactar un plan de instrucción general y universitaria. Jacques fue nombrado jefe de estudios del Colegio Nacional de Buenos Aires, que era dirigido por el padre Eusebio Agüero quien estaba a cargo del Colegio Eclesiástico, antiguamente Colegio de Ciencias Morales. En su amplísima memoria, Jacques muestra todo su saber analizando el rol de los alumnos, del profesor, cómo son los contenidos, cuántos años se cursan, el plan de estudios en general muestra un marcado carácter humanista y enciclopedista, la educación secundaria es vista como preparatoria para las carreras universitarias. Los Colegios Nacionales se fueron fundando en las capitales provinciales, algunos sobre la base de otras instituciones como en Buenos Aires y Tucumán o se fundaron en aquellos lugares donde no había colegios secundarios. En 1863 se crea el Colegio Nacional de Buenos Aires, en 1865 se crean los colegios de Tucumán, Mendoza, San Juan, Catamarca y Salta; en 1869 llega el turno de Jujuy, Corrientes, Santiago del Estero y San Luis; luego se fundan colegios en La Rioja (1871) y Rosario (1874). ${ }^{5}$

Según Juan Carlos Tedesco (2009), estas instituciones fueron creadas para la formación de los hijos de las élites provinciales, y aunque debatidas sus tesis $^{6}$, se orientaron a la creación de una élite política de ideología nacional que pudiera, de esta manera, superar las diferencias entre el litoral, la provincia de Buenos Aires y el Estado Nacional que se estaba gestando. En esta concepción acera de formación de élites locales bajo los "ideales de la Nación", se puede comprender la importancia política de mantenerlos, pese a que en muchas regiones contaban con pocos alumnos. Siguiendo a Susana Schoo (2011),

Esta hipótesis explicaría por qué se los sostuvo a pesar de su baja matrícula, escasez de profesores y resultados de la enseñanza, especialmente en las décadas de 1860 y 1870. ¿Por qué ahora, en la década de 1880 primero se concedió a la Provincia un colegio nacional para luego negárselo con fundamentos poco sólidos? (p. 138). 
Por un lado, la autora sostiene que una respuesta posible ante ese interrogante haya sido que aquellos que habían estudiado en esos colegios y ya ocupaban cargos públicos, eran plenamente conscientes que el ingreso de otros sectores sociales a los Colegios y las Universidades traería graves consecuencias. ${ }^{7}$ Sin embargo, como ya mencionamos, la provincia de Buenos Aires se encontraba en plena lucha con las autoridades nacionales por lo que "la negativa a la creación del colegio nacional puede ser entendida en el marco de una disputa mayor entre distintas fracciones del poder que manifestaban sus conflictos en diversos temas de mayor o menor envergadura." (Schoo, 2011: 139). Claro que la educación secundaria no estuvo exenta de la situación tirante. En este plano de tensiones entre Buenos Aires y el Estado Nacional, se comprende mejor el porqué de la negativa de Roca a crear un Colegio Nacional en la capital de la Provincia de Buenos Aires. La élite nacional entendió que el modelo de colegios se expandió rápidamente y que el acceso a los cargos públicos seguía en manos de un grupo cerrado de quienes hacían la política.

Finalmente, la Provincia implantó el Colegio secundario en La Plata a través del decreto del gobernador Carlos D'amico, el 25 de febrero de 1885 . El mismo se instaló en un local ubicado en la esquina de calle 9 y 47, adoptó el plan de estudios del Colegio Nacional de Buenos Aires (conforme a la Ley de Libertad de Enseñanza de 1878, y su fundación fue refrendada por el gobierno nacional dos meses más tarde. De esta manera, el gobernador ponía fin a la situación irregular en cuanto a la educación secundaria en la provincia. Un año más tarde el colegio se muda a un edificio de propiedad provincial en la Avenida 51 entre calles 17 y 18 (hoy Ministerio de Salud). Por otro lado, se designa rector al italiano Matias Calandrelli, y una nómina de docentes para empezar las clases lo más pronto posible. Se otorgaba el título de bachiller y los alumnos podían continuar los estudios universitarios pero para ello, al no tener la ciudad una Universidad, sí o sí tenían que trasladarse a la ciudad de Buenos Aires.

Para el caso de la Educación Física en la red de Colegios Nacionales, la misma fue fluctuando desde el plan fundacional donde ni siquiera es citada para luego ir ganando terreno en los planes de estudios. Aparece como gimnasia, calistenia, ejercicios físicos, juegos y recreos, etc., pero siempre con un lugar marginal frente a las disciplinas "intelectuales". El tema es intensamente estudiado por quien fuera Inspector de Educación Física, el Dr. Enrique Romero Brest, figura indiscutible de la Educación Argentina desde los inicios del 1900 hasta la tercera década del siglo XX. En su informe ante el Censo general de Educación del año 1909, el Inspector sostiene que la cultura física está totalmente descuidada ya que no suele tener el lugar que le corresponde. La ambigüedad con la cual se trataba a la Educación Física (en plena gestación) era tal que incluso en 1895 se la totalmente y se la reemplaza por ejercicios militares. En su informe trabaja tres etapas, la primera que va de 1863 hasta $1898^{8}$, aquí resaltamos dos Colegios donde los ejercicios físicos y los juegos al aire libre tuvieron un gran desarrollo y le dieron la importancia que se merecían. Me refiero en primer lugar al Colegio Nacional de Corrientes, dirigido por Santiago Fitz Simón; y el Colegio Nacional de Concepción del Uruguay, con el doctor José Benjamín Zubiaur a la cabeza. En el caso de Corrientes, según Jorge Saraví Riviere (2012), el rector contrata un docente inglés llamado Tomas Reeve para dicar la clase de "ejercicios físicos". Aquí se 
desarrollan los juegos ingleses al aire libre, los sports, como el football, el lawn tennis y el rugby, importando los ideales desde las Public Schools inglesas como Eton, Winchester y Rugby.

En el caso del Colegio del Uruguay, es destacado el protagonismo del rector José Benjamín Zubiaur, quien en 1892 contrata al Dr. Joseph Gybbon Spilsbury para las clases de "gimnasia". Aquí se promueven los ejercicios al aire libre y las excursiones escolares además de comprar materiales y mobiliario para la práctica de natación, remo, tenis y gimnasia de aparatos.

\section{La Plata: Colegio y (re)fundación de la Universidad}

Luego de la fundación del Colegio Provincial de La Plata, que por gestiones del gobernador Máximo Paz fue transferido a la Nación en 1887 haciéndose efectivo un año después, la provincia crearía una Universidad bajo su órbita. Inspirado por un proyecto que encabezó Rafael Hernández (hermano del autor del Martín Fierro, José Hernández), en 1889 se crea la Universidad de La Plata que, por los vaivenes económicos y políticos, no pudo abrir sus puertas hasta el año 1897. La decisión de abrir una universidad fue ampliamente saludada por los intelectuales de la región y los jóvenes estudiantes del Colegio, ahora nacional. La Universidad tomó los estatutos de la de Buenos Aires y se regiría por la ley de enseñanza superior. Cabe destacar que la Universidad no cuenta en sus inicios con otros órganos científicos que ya funcionaban en la ciudad como la Facultad de Agronomía y Veterinaria, el Museo de Ciencias Naturales, el Observatorio Astronómico y la Biblioteca Pública de la provincia. Estos estandartes de la ciencia serían tenidos muy en cuenta por Joaquín V. González para su proyecto de universidad nueva. Superadas las crisis económicas, y por decreto del gobernador Guillermo Udaondo, en 1897 comenzaron las clases universitarias siendo su primer rector el Dr. Dardo Rocha. La universidad funcionó a paso errante debido a la baja matrícula, oferta poco atractiva y el poco reconocimiento que tenían sus títulos frente a los que expedía la Universidad de Buenos Aires, por lo que rápidamente entró en decadencia.

Frente a ello, en 1905 el Ministro de Justicia e Instrucción Pública Joaquín V. González ${ }^{9}$, estudió a fondo la situación de la universidad platense, encontraba en La Plata la ciudad ideal para llevar adelante su atractivo plan. El mismo se basó en una serie de acuerdos anteriores por los que la provincia había cedido a la nación la Facultad de Agronomía y el Observatorio. González propone también la cesión a la nación de la Biblioteca Pública, el Museo de Ciencias Naturales, el edificio del Banco Hipotecario y lo que quedaba de la agonizante Universidad de La Plata. Otro de los acuerdos fue el pasaje a la nación de 18 hectáreas para la construcción de un Colegio Nacional modelo, hoy ubicado en la Avenida 1 entre calles 47 y 50.

El Ministro riojano, valiéndose de sus estudios de los sistemas universitarios europeos y de los Estados Unidos, esbozó una memoria en la cual expuso su proyecto, ambicioso pero concreto de reorganizar los institutos científicos platenses para crear una universidad con una matriz nueva, distinta a las ya existentes como Córdoba y Buenos Aires. Su plan de enseñanza 
abarcaba todo el sistema educativo ya que relacionaba las escuelas primarias y la universidad a través del Colegio Nacional modelo, que pasó a depender de la Universidad en 1907. A su vez, le imprimía un nuevo sesgo científico, racional y experimental a la nueva casa de estudios, promoviendo la extensión universitaria a través de las conferencias diurnas y nocturnas sobre diversos temas, motivando los intercambios de estudiantes y docentes con otras universidades del mundo, equipando los laboratorios de investigación, contratando profesiona-les reconocidos del mundo intelectual incluso extranjeros, tanto para dirigir las cátedras como para regir los equipos de investigación.

Sin embargo, el gran proyecto de González dentro de la Universidad era el Colegio Nacional, al que le dedicó muchas páginas en su proyecto y mucho énfasis en sus discursos. En esta Memoria se refiere a la forma de vida a adoptar en la institución, a la cual se refiere como vida universitaria, que tiene un valor educativo en sí mismo y se manifiesta en la relación entre alumnos entre ellos y entre alumnos y maestros. Critica con lucidez la falta de vida higiénica y física en las casas de estudio que respondieron siempre a la tradición conventual, de claustros cerrados, sin amplias avenidas y parques, algo que destacaba a La Plata con su epicentro en el paseo del Bosque y su imponente avenida de eucaliptus y robles.

La construcción del Colegio fue encargada a los ingenieros Miguel Olmos y Carlos Massini en 1905 ambos del Ministerio de Obras Públicas de la Nación. La piedra fundacional se colocó el 6 de septiembre de ese año y en su discurso González, todavía Ministro de Justicia e Instrucción Pública, exponía las virtudes del Internado Moderno que contaría el Colegio. Regiría el sistema tutorial, en el cual cada uno de los internados (se proyectaron cuatro pero solo se construyeron dos), se alojarían entre 25 y 40 alumnos dirigidos por un tutor encargado. Se proyectó un edificio central monumental en el frente de la Avenida 1 de 134 metros, tres pisos de alto; un cuerpo destinado a los laboratorios de física y química, ciencias naturales y museos; y el gimnasio de estilo griego, con amplios terrenos para juegos atléticos, piscina de natación, gimnasia metódica, tiro al blanco, pelota, carreras a pie, bicicleta, disco, fútbol, y demás actividades de los sistemas de ejercicios físicos. La construcción del predio comenzó en 1905 inaugurándose todo el complejo para el año lectivo de 1910. Los internados $N^{\circ} 1$ y № 2 (actuales Facultades de Ingeniería y de Ciencias Exactas) se situaron a la derecha del Colegio, sobre la Avenida 1 hacia calle 47.

Punto fundamental sobre la vida cotidiana del Colegio, el internado moderno abierto, social y libre se contrapone al antiguo que era característico por ser de tipo monacal, hospitalario, claustral el cual posee régimen de horarios de entrada, salida, vigilancia, etc. El nuevo internado suprime el dormitorio común "foco de abusos y de vicios" como se refería González a los internados típicos de Europa; según él, "la habitación independiente ofrece aún otras ventajas, así del punto de vista moral como de la higiene. Los alumnos tendrán la libertad para hacer su toilette íntima, sin provocar miradas o gestos indecorosos y sin verse expuestos a soportarlos." ${ }^{10}$ (González, 1935: 52-53). Por último, la convivencia de los alumnos con jóvenes de su misma edad y con los académicos dedicados tanto a la enseñanza como a la investigación, es lo 
que caracteriza este tipo de internado y a la comunidad universitaria en general. Por otro lado, en palabras de Miguel Olmos:

El gimnasio lo constituyen dos grandes patios de juegos atléticos con canchas para foot-ball, carreras, velódromo, etc., una pileta de natación de 20 por 60 metros de largo, una cancha de pelota para cinco juegos á mano y un á cesta, el tiro y un gimnasio cubierto, faltando las gradas y locales para la concurrencia á los matchs. El gimnasio cubierto es una verdadera joya de órden dórico modernizado, podría decirse que es un templo de estilo griego levantado á la educación física. ${ }^{11}$ (Olmos, 1907: 201-202).

En 1910 se inauguran los cursos en los nuevos edificios del Colegio Nacional. Previo a ello, en 1906 Joaquín V. González renuncia a su cargo de Ministro para ser elegido como el nuevo Presidente de la Universidad Nacional de La Plata; ejerce la presidencia luego de ser reelegido tres veces hasta 1918, cuando entrega el cargo a Rodolfo Rivarola.

\section{A modo de cierre}

A grandes rasgos se hizo un recorrido histórico acerca de la ciudad de La Plata, la creación del Colegio, creado bajo la órbita provincial, pasando por la nacionalización hasta llegar a depender de la Universidad Nacional de La Plata. En otros trabajos, así como también en mi tesis de Licenciatura en Educación Física en curso, se desarrolla más a fondo la propuesta central acerca del Internado moderno y cómo concibió a la Educación Física el Ministro Joaquín V. González.

El mismo dotó de gran importancia a los deportes y juegos al aire libre, la concepción de vida higiénica, la contemplación al aire libre, incluso la utilización del canal navegable de Ensenada, en el cual aspiraba a ver regatas universitarias al estilo de Cambridge y Oxford.

Es necesario destacar el rol que tuvo el Ministro riojano ya que, gracias a su experiencia y conocimiento fundó la Universidad Nacional de La Plata imprimiéndole una impronta científica y experimental comunicando el conocimiento, incentivando también los juegos y deportes en el Colegio y sus Internados.

\section{Referencias}

Blanco, M. M. "Historia del Colegio Nacional de La Plata." En Labor del Centro de Estudios Históricos. (1940-1941).

Gonzalez, J. V. (1935). Obras Completas. Tomo XIV. Imprenta Mercatali, Buenos Aires. 
Olmos, M. (1907). "Colegio nacional de La Plata. Proyecto del Ingeniero Olmos." En Revista La Ingeniería, n. 11 (13). pp. 199-204. Centro Nacional de Ingenieros, Buenos Aires.

Sabato, H. (2012). Historia de la Argentina, 1852-1890. Siglo XXI editores, Buenos Aires.

Schoo, S. (2011). "La educación secundaria en Buenos Aires: De la universidad provincial al Colegio Nacional." En Revista Archivos de Ciencias de la Educación. № 5. Departamento de Ciencias de la Educación, Facultad de Humanidades y Ciencias de la Educación, Universidad Nacional de La Plata.

Tedesco, J. C. (2009). Educación y sociedad en la Argentina (1880-1945). Siglo XXI, Buenos Aires.

Troisi, J. (2006). Dardo Rocha: el último porteño. Instituto Cultural de la Provincia de Buenos Aires, La Plata.

Vallejo, G. (2007). Escenarios de la cultura científica argentina. Ciudad y universidad: 1882-1955. Consejo Superior de Investigaciones Científicas, Madrid.

\section{Notas}

${ }^{1}$ La ciudad de La Plata cuenta con calles numeradas en vez de tener nombres como se acostumbra en muchas ciudades de Buenos Aires y la Argentina. Por un lado, el casco histórico contempla la Avenida 122 en forma decreciente hasta la calle 115, luego la Avenida 1 en forma creciente hasta la Avenida 31, y en otro "lado" del cuadrado la numeración continúa con la Avenida 32 hasta la Avenida 72.

${ }^{2}$ Nieto del político y militar Juan Manuel de Rosas, gobernador de la Provincia de Buenos Aires en los períodos $1829-1832$ y $1835-1852$.

${ }^{3}$ Nacido en Francia en 1848, llega a la Argentina de modo errático a los 18 años. Luego de un paso por San Antonio de Areco se instala en Tucumán, dictando clases en la Escuela Normal (dirigiéndola de 1878 a 1881) y en el Colegio Nacional. En 1884 se asienta en Buenos Aires donde funda el diario Sud-América, ocupa el cargo de Inspector de Enseñanza Secundaria y Normal, y es designado Jefe de la Biblioteca Nacional, cargo que ocupó hasta su muerte en 1929. Fue un gran intelectual y literato de su época, teniendo su opinión un lugar destacado entre los intelectuales contemporáneos.

${ }^{4}$ Según Miguel Cané (1992) nace en París en 1813, estudia en la Escuela Normal Superior y luego tiene una destacada participación intelectual y como profesor en el Colegio Luis Le Grande. Funda una revista y participa en los movimientos revolucionarios de 1848. Tres años más tarde se exilia en Montevideo y luego se traslada a la Argentina recorriendo el Chaco, Santiago del Estero y Tucumán por encargo del presidente Urquiza. Se establece en Tucumán dirigiendo el Colegio de San Miguel hasta que Mitre lo llama a Buenos Aires en 1863 para la confección del Plan de Estudios del Colegio Nacional, el cual dirige hasta su muerte en 1865. Para ampliar véase Cané, M. (1992). Juvenilia. Centro Editor de América Latina, Buenos Aires.

${ }^{5}$ En 1854 ya habían pasado a la tutela nacional el Colegio de Monserrat de la ciudad de Córdoba (junto con su Universidad) y el Colegio de Concepción del Uruguay de Entre Ríos que había sido fundado por Justo José de Urquiza en 1849.

${ }^{6}$ Martín Legarralde concuerda con Tedesco acerca de la función eminentemente política de la educación en este tipo de instituciones, aunque no le parece tan claro cómo esa función política de articulación de intereses entre las élites locales y la ideología de la clase dirigente porteña se llevó a cabo. Por otro lado en su mismo trabajo sostiene que "las élites preexisten a los colegios nacionales"; las mismas se estructuraron políticamente en tres sectores: Buenos Aires, litoral e interior, "configurando un panorama conflictivo en el que difícilmente una institución pudiera establecer un consenso en torno a la ideología porteña." (Legarralde, 1999: 42). Por último, y apoyándose en Botana $(1992,1993)$, durante el período abarcado por 
Tedesco (desde 1863 a 1890) "el poder central ha cambiado de manos". Son ahora las provincias del interior las que manejan los mecanismos de control de las sucesiones presidenciales. Por lo que el sector mitrista, que dio origen a los Colegios Nacionales, rápidamente perdió protagonismo en la disputa por el poder central de la nación. Para ampliar véase Legarralde, M. (1999). "La fundación de un modelo pedagógico: los colegios nacionales entre 1862 y 1887." En Revista Propuesta Educativa. n. 21, pp. 38-43.

${ }^{7}$ El acceso de sectores medios a los Colegios Nacionales y las Universidades pondría en jaque al sistema político oligárquico característico de los '80. Ello llevaría a la fractura del Partido Autonomista Nacional y la creación de la Unión Cívica, luego de la Revolución del Parque, perpetrada por Lisandro de Latorre, Leandro Alem, Bartolomé Mitre, entre otros, llevando a la caída al presidente Juárez Celman en 1890 y la llegada al poder del vicepresidente, aliado de Roca, Carlos Pellegrini.

${ }^{8}$ En 1898 se reglamenta la obligatoriedad de los ejercicios físicos en todos los años del plan de estudios de los Colegios Nacionales y las Escuelas Normales a través de un decreto de la presidencia, refrendado por el Ministro Belaustegui.

9 Nacido en Nonogasta, La Rioja en 1863, tuvo una brillante carrera política e intelectual ocupando diferentes cargos políticos como diputado, senador, Ministro de Roca y del presidente Quintana. Tuvo destacada actuación parlamentaria en la elaboración del primer código de trabajo como también del código electoral. Luego de ser Presidente de la UNLP entre 1906 y 1918, muere en 1923 siendo senador nacional por su provincia. El Congreso de la Nación editó en 1935 todas sus obras en 25 tomos.

${ }^{10}$ La cursiva pertenece al original.

${ }^{11}$ La ortografía y la cursiva pertenecen al original. 